\title{
Strain Imaging by Speckle Tracking for the Assessment of Diastolic Dysfunction in Beta-Thalassemia Major Patients
}

Mufide Okay', [MD]

ORCID: 0000-0001-5317-0597

Cem Coteli², [MD]

ORCID: 0000-0001-5520-7103

Sule Unal ${ }^{3},[\mathrm{MD}]$

ORCID: 0000-0002-3083-8547

Tuncay Hazirolan ${ }^{4}$, [MD]

ORCID: 0000-0001-8905-1768

Erdem Karabulut ${ }^{5},[\mathrm{MD}]$

ORCID: 0000-0002-7811-8215

Necla Ozer', [MD]

ORCID: 0000-0001-7914-0169

Fatma Gumruk ${ }^{3}$, [MD]

ORCID: 0000-0001-5102-0624

Nilgun Sayinalp ${ }^{6},[\mathrm{MD}]$

ORCID: 0000-0002-4782-896X

'Ministry of Health, Eskisehir City Hospital, Department of Hematology, Eskişehir, Turkey.

${ }^{2}$ Hacettepe University School of Medicine, Department of Cardiology, Ankara, Turkey.

${ }^{3}$ Hacettepe University School of Medicine, Department of Pediatric Hematology, Ankara, Turkey.

${ }^{4}$ Hacettepe University School of Medicine, Department of Radiology, Ankara, Turkey.

${ }^{5}$ Hacettepe University School of Medicine, Department of Biostatistics, Ankara, Turkey.

${ }^{6}$ Hacettepe University School of Medicine, Department of Hematology, Ankara, Turkey.

Corresponding Author: Mufide Okay

Eskisehir City Hospital, Department of Hematology 26080, Eskisehir, Turkey.

E-mail:mufide_okay@yahoo.com

This research did not receive any specific grant from funding agencies in the public, commercial, or not-forprofit sectors.

https://doi.org/10.32552/2021.ActaMedica.533

\section{n ABSTRACT Con}

Objectives: Strain imaging is a novel promising echocardiographic technique and strain imaging by speckle tracking has been reported recently in a few studies as a promising tool to detect early changes of myocardial deformation that could be related to myocardial iron over load in patients with $\beta$-thalassemia major. In this study, we investigated strain images in comparison with the cardiac $\mathrm{T}^{*}$ magnetic resonance imaging results.

Materials and Methods: A total of 45 patients from a single institution's pediatric and adult hematology centers were evaluated with strain echocardiography and $\mathrm{T} 2^{*}$ magnetic resonance imaging at concomitant out-patient visits. We grouped patients according to cardiac $\mathrm{T2}^{*}$ magnetic resonance imaging results: $<20 \mathrm{~ms}$ (Group 1, cardiac iron loading present) ( $n=12)$ and $\geq 20 \mathrm{~ms}$ (Group 2, no clinically significant cardiac iron loading) $(n=33)$.

Results: The median global longitudinal strain was measured as $-19.9 \%$ (range $-27.1 \%$ to $-12 \%$ ) in Group 1 and $-20.7 \%$ (range $-26.5 \%$ to $-12.8 \%$ ) in Group $2(p=0.37)$. Using receiver operator curve analysis, global longitudinal strain value at a cutoff point of $-20.45 \%$ in the ruling out $2^{*}<20$ ms with a sensitivity of $83 \%$ and a specificity of $54 \%$ was obtained.

Conclusion: In conclusion, although $\mathrm{T} 2 *$ magnetic resonance imaging is the gold standard method, strain imaging echocardiography can be used for predicting cardiac iron accumulation, after validations were obtained in larger sample sizes.

Keywords: Thalassemia, strain imaging, diastolic dysfunction, cardiac, echocardiography

Received: 11 November 2020, Accepted: 4 December 2020

Published online: 5 February 2021 


\section{INTRODUCTION}

Cardiac iron accumulation is the most important cause of mortality for $\beta$-thalassemia major (BTM) patients [1]. Increased iron absorption due to ineffective erythropoiesis and chronic blood transfusions are the cause of iron accumulation for these patients [2] . Liver, heart and endocrine organs are the major sites of iron accumulation [2, 3].

Cardiac $\mathrm{T}^{*}$ magnetic resonance imaging (MRI) is the gold standard, non-invasive and validated method for assessment and follow-up of myocardial iron overload indirectly [4]. Cardiac T2* value below $10 \mathrm{~ms}$, is indicative for severe iron accumulation and $\mathrm{T}^{*}$ values above $20 \mathrm{~ms}$ indicates the absence of clinically significant iron accumulation. On the other hand, range of $\mathrm{T}^{*}$ value between $10-20 \mathrm{~ms}$ indicates cardiac iron loading $[5,6]$. However, access to cardiac T2* MRI is still limited in many centers [7].

Conventional echocardiography is suggested to be performed annually for BTM patients when they reach the age of ten. However, conventional echocardiography can only detect systolic dysfunction when iron overload reaches its severe levels in the late period of disease [8,9]. Strain imaging, also known as deformation imaging, is a novel promising echocardiographic technique which allows assessment of regional myocardial function [10]. Strain imaging can be done by using tissue doppler imaging or speckle tracking [11]. Speckle tracking is a recent echocardiographic approach which is a post-processing computer algorithm [10]. Strain imaging by speckle tracking was reported as a promising tool to detect early changes of myocardial deformation that could be related to myocardial iron over load in patients with BTM [12].

In this study, we aimed to compare strain echocardiography parameters and cardiac T2* MRI results of BTM patients form a single center.

\section{MATERIALS and METHODS}

\section{Patient Population}

A total of 45 patients from a single institution of pediatric and adult hematology centers with BTM diagnosis were evaluated with strain echocardiography and T2* MRI at concomitant outpatient visits. The echocardiographic evaluations and $2^{*} \mathrm{MRI}$ measurements were performed with Local Ethical Committee approval (GO-19386 Number) and informed consent were obtained from all patients/parents (for under 18 years old patients). Only the BTM patients who were on regular transfusion programme were included in this study. Patients with arrhythmias, hypertension, moderate to severe valvular diseases and congenital heart diseases were excluded. Mean serum ferritin levels of the last 12 months before assessment was also recorded.

Myocardial iron load was assessed using 1.5 Tesla scanner (Siemens Aera, Erlangen, Germany) for all patients. "Multi Echo Black Blood Gadolinium Related Enhancement (GRE)" images were obtained during the mid-diastolic phase of cardiac cycle to calculate $\mathrm{T}^{*}$. Myocardial T2* were measured from mid-ventricular septum on short axis view. All cardiac T2* MRI assessments were evaluated by the same radiologist. Patients were grouped according to cardiac T2* MRI measurements: $<20$ ms (Group 1) and $\geq 20 \mathrm{~ms}$ (Group 2).

\section{Echocardiographic Evaluation}

Conventional transthoracic echocardiography was performed using GE Vivid E9 3D Echo by the same cardiologist who was blinded to the clinical data. Two-dimensional image examination and chamber quantification were performed in parasternal long- and short-axis views and apical four-, twochamber and long axis views, according to the recommendations of the European Association of Cardiovascular Imaging (EACVI) [13]. Conventional left and right ventricular systolic function parameters which are left ventricle end diastolic diameter (LVEDD), left ventricle ejection fraction (LVEF), end-diastolic mid-right ventricle diameter (RVEDD) and tricuspid annular systolic excursion (TAPSE) were calculated.

Left ventricular diastolic function was evaluated with mitral inflow velocities [E wave, A wave, E/A ratio, deceleration time of $E$ wave (DT)], tissue doppler velocities of mitral annular septal and lateral wall (e' septal, e' lateral), isovolumetric relaxation 
time (IVRT). Left ventricle filling pressure was estimated with E wave / mean e' ratio and the apical four chamber, apical two chamber and apical long axis views were recorded for post procedural strain analysis. All patients were informed and consent was gotten to record echocardiographic views. All views were recorded from five consecutive beats at the end expiration and frame rate was set to be more than $60 \mathrm{fps}$. Left ventricle global longitudinal strain (GLS) values were calculated with GE EchoPAC Clinical Workstation Software. Strain values were expressed as the negative percentage of values. All echocardiographic evaluation for all patients was performed by the same operator.

\section{Statistical Analyses}

Independent sample $t$ test and Mann Whitney $U$ tests were used for normally and non-normally distributed variables, respectively. Variables were described by using mean \pm standard deviation (SD) for normally distributed variables and the median (minimum-maximum) value for non-normally distributed variables. The receiver operating characteristic (ROC) curve was used to obtain the optimal cut-off for global longitudinal strain value in diagnosing cardiac involvement by considering T2* MRI result as a gold standard test. Statistical significance level was considered as $p<0.05$. The data were analyzed with IBM SPSS Statistics for Win ver. 23 (IBM Corporation, Armonk, NY, USA).

\section{RESULTS}

The baseline patient's characteristics are presented in Table 1. Median age of patient population was 31.6 (minimum-maximum: 21.5 - 47.1). The iron chelators that the patients were on during evaluation were summarized in Table $1.66 .6 \%$ and $66.7 \%$ of Group 1 and 2 patients were on deferasirox as iron chelation therapy, respectively.

\section{Echocardiography and Doppler Findings}

Left ventricle ejection fraction was measured as $60.58 \%$ and $64.26 \%$ in Group 1 and 2, respectively $(p=0.01)$.

Left ventricular diastolic function parameters were 1.7 and 1.5 for $E / A$ ratio $(p=0.01), 8.9$ and 8.2 for $E /$ septal e' ratio $(p=0.04), 6.75$ and 6.3 for E/lateral $e^{\prime}$ ratio $(p=0.14$ ) in Group 1 and 2, respectively (Table 2).

Mean DT values were $176.83 \mathrm{~ms} \pm 37.29 \mathrm{~ms}$ and $169.46 \mathrm{~ms} \pm 28.91 \mathrm{~ms}$ in group 1 and 2 , respectively $(p=0.5)$. IVRT times were 80 and 83 in group 1 and 2 , respectively $(p=0.66)$.

\section{Left Ventricle Global Longitudinal Strain}

When we compared the groups for speckle imaging, the median average global longitudinal strain values were $-19.9 \%(-27.1 \%--12 \%)$ for group 1 and $-20.7 \%(-26.5 \%--12.8 \%)$ for group 2 ( $p=0.37)$.

Table 1. Demographic characteristics of the groups.

\begin{tabular}{|l|c|c|c|}
\hline & Group 1 $(\mathrm{n}=12)$ & Group 2 $(\mathrm{n}=33)$ & $p$ \\
\hline Age (mean \pm SD) (years) & $30.6 \pm 5.74$ & $33.2 \pm 6.09$ & 0.19 \\
Gender (female percentage) & $8 / 12(66.7 \%)$ & $16 / 33(48.5 \%)$ & 0.28 \\
\hline Median serum ferritin (range) (pmol/L) & $2458.22(530.29-7612.84)$ & $1184.17(156.62-17888.37)$ & 0.03 \\
\hline
\end{tabular}

SD: standard deviation

Table 2. Comparison of the echocardiographic variables between the groups.

\begin{tabular}{|l|c|c|c|}
\hline & Group 1 $(\mathrm{n}=12)$ & Group 2 $(\mathrm{n}=24)$ & $p$ \\
\hline LVEF (mean) (\%) & $60.58 \pm 4.85$ & $64.26 \pm 4.18$ & 0.01 \\
DT (mean) (ms) & $176.83 \pm 37.29$ & $169.46 \pm 28.91$ & 0.50 \\
E/A ratio & 1.7 & 1.5 & 0.01 \\
IVRT & 80 & 83 & 0.66 \\
E/e' ratio (septal wall) & 8.9 & 8.2 & 0.04 \\
E/e' ratio (lateral wall) & 6.75 & 6.3 & 0.14 \\
Global longtitudinal strain & $9(75 \%)$ & $20(83.3 \%)$ & 0.55 \\
\hline Global longtitudinal strain $\geq-18 \%$ & $3(25 \%)$ & $4(16.7 \%)$ & \\
\hline
\end{tabular}

LVEF: left ventricle ejection fraction, DT: deceleration time of E wave, E/A: E wave/A wave, IVRT: isovolumetric relaxation time. 
Using receiver operating characteristic (ROC) curve, global longitudinal strain value $-20.45 \%$ predicted a T2* value of $<20$ ms with a sensitivity of $83 \%$ and a specificity of $54 \%$. The area under the ROC curve (AUC) was 0.609 (95\% Cl 0.406 to 0.813 ) (Figure 1).

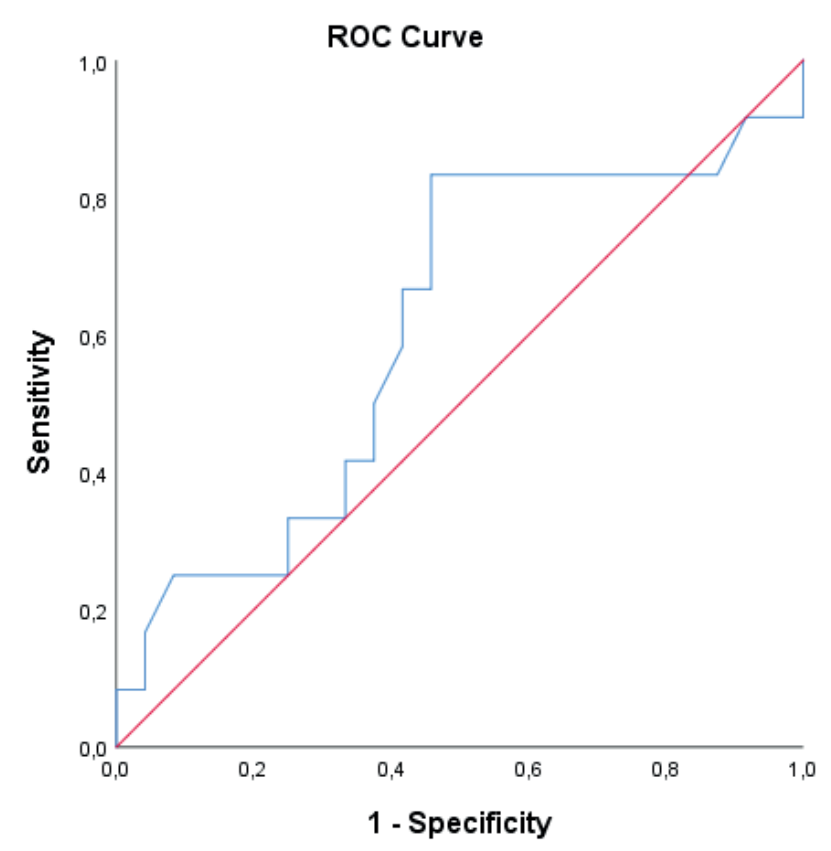

Diagonal segments are produced by ties.

\begin{tabular}{|c|c|c|c|c|}
\hline \multirow[b]{2}{*}{ Area } & \multirow[b]{2}{*}{ Std. Error ${ }^{\mathrm{a}}$} & \multirow[b]{2}{*}{$p$} & \multicolumn{2}{|c|}{$95 \% \mathrm{Cl}$} \\
\hline & & & Lower & Upper \\
\hline 0,609 & 0,104 & 0,290 & 0,406 & 0,813 \\
\hline
\end{tabular}

Figure 1. Receiver operating characteristic curve for the global longitudinal strain levels by considering $\mathrm{T} 2^{*}<20 \mathrm{~ms}$ as a gold standard

\section{DISCUSSION}

Diastolic dysfunction usually develops before systolic dysfunction in patients with BTM.

Myocardial iron accumulation makes early diastolic dysfunction without affecting the relaxation of the left ventricle. In the later period, systolic dysfunction develops as dilated cardiomyopathy. With the early detection of diastolic dysfunction, changes can be made with iron chelation therapy, and expected systolic dysfunction can be prevented [14]. In our study, left ventricle diastolic functions were evaluated with transmitral doppler and tissue doppler values. To our knowledge, E/e' ratio is an important predictor for increasing the left ventricle diastolic pressure. In our series, E/ septal e' ratio was significantly higher in group 1 and this finding showed that diastolic dysfunction was more prominent in group 1 consistent with Cardiac MRI findings.

Cardiac T2 ${ }^{*} \mathrm{MRI}$ is the gold standard tool for the assessment of myocardial iron deposition in BTM patients. However, T2* MRI is not available in every center which follows BTM patients. Other disadvantages of MRI are its contraindication in the presence of cardiac pacemaker and claustrophobia. Echocardiographic and strain assessment may be an alternative diagnostic tools to T2* MRI [15]. In the previous study conducted by Garceau P et al., showed that the ejection fractions were found as $59 \%$ and $64 \%$ in low T2* (high cardiac iron load) and normal T2* (normal cardiac iron load) groups, respectively. They also found no difference in two groups in terms of strain parameters except for E/A ratio [15]. Our results are compatible with the results of Garceau et al. On the other hand, Garceau et al. showed decrease in global longitudinal strain values in low T2* BTM patients compared to normal T2* group. In our study, although global longitudinal strain was found to be low in the low T2* group, no statistically significant difference was found.

In another study, peak atrial 2D strain was found as an early index of left ventricular diastolic dysfunction in BTM patients [16]. Atrial strain values were examined in this study with the $2 \mathrm{D}$ strain and there was no significant difference in longitudinal strain among the groups [16]. In our study, ventricular global longitudinal strain values were evaluated. Although the specificity was low, we found the cut-off value of global longitudinal strain as $-20.45 \%$ to predict high iron overload (low T2* MRI) and this was different from other studies $(-17 \%$ and $-18.5 \%)[15,17]$.

In a recent study, global longitudinal strain was found as an early predictor for diastolic dysfunction in 122 thalassemia major and intermedia patients [17]. Pizzino et al. also showed that GLS $-19.5 \%$ can be predictive for myocardial iron overload [18]. Our study was compatible with the results of these studies. However, in our study, the cut-off value of global longitudinal strain was found to be lower, interestingly.

It is important to realize the limitations of this study. First of all, our study population was small 
due to rarity of the BTM. However, we conducted 45 patients and this was enough to get significant biostatistical analysis. Secondly, measurement of iron burden was made only in the mid ventricular septum. Speckle tracking imaging is a segmental technique with a global value and physicians can compare global longitudinal strain in the mid ventricular septum. Finally, in the future, next studies may compare the treatment efficacy with speckle tracking and strain parameters.

In conclusion, although $\mathrm{T} 2{ }^{*} \mathrm{MRI}$ is the gold standard method to determine the cardiac iron load, strain imaging echocardiography can be used for predicting cardiac iron accumulation in thalassemia patients at $2^{*} \mathrm{MRI}$ absent centers. The patients who have impaired global longitudinal strain value (>-20.45\%) might be suggested for evaluation with T2* MRI. Strain imaging by speckle tracking and left ventricular diastolic function parameters might be used as a screening tools in such centers that do not have T2*MRI.

\section{CONFLICT Of INTEREST STATEMENT}

The authors of this paper have no competing interests'.
[1] Aydinok Y. Thalassemia. Hematology 2012; 17 Suppl 1: S28-31.

[2] Aydinok $\mathrm{Y}$, Porter JB, Piga A, et al. Prevalence and distribution of iron overload in patients with transfusiondependent anemias differs across geographic regions: results from the CORDELIA study. Eur J Haematol 2015;95(3): 244-53.

[3] Hoffbrand AV, Taher A, Cappellini MD. How I treat transfusional iron overload. Blood 2012;120(18): 3657-69.

[4] Gorcsan J, Tanaka H. Echocardiographic assessment of myocardial strain. Journal of the American College of Cardiology 2011; 58(14): 1401-13.

[5] Carpenter JP, Roughton M, Pennell DJ. International survey of $\mathrm{T}^{*}$ cardiovascular magnetic resonance in betathalassemia major. Haematologica 2013; 98(9): 1368-74.

[6] Meloni A, De Marchi D, Positano V, et al. Accurate estimate of pancreatic T2* values: how to deal with fat infiltration. Abdominal Imaging 2015; 40(8): 3129-36.

[7] Aydinok Y, Oymak Y, Atabay B, et al. A National Registry of Thalassemia in Turkey: Demographic and Disease Characteristics of Patients, Achievements, and Challenges in Prevention. Turk J Haematol 2018;35(1): 12-8.

[8] Kremastinos DT, Tsiapras DP, Tsetsos GA, et al. Left ventricular diastolic Doppler characteristics in betathalassemia major. Circulation 1993;88(3): 1127-35.

[9] Kostopoulou AG, Tsiapras DP, Chaidaroglou AS, et al. The pathophysiological relationship and clinical significance of left atrial function and left ventricular diastolic dysfunction in beta-thalassemia major. Am J Hematol 2014; 89(1): 13-8.

[10] Gorcsan J, 3rd, Tanaka H. Echocardiographic assessment of myocardial strain. Journal of the American College of Cardiology 2011; 58(14): 1401-13.

[11] Collier P, Phelan D, Klein A. A Test in Context: Myocardial Strain Measured by Speckle-Tracking Echocardiography. Journal of the American College of Cardiology 2017; 69(8): 1043-56.
[12] Gupta A, Kapoor A, Phadke S, et al. Use of strain, strain rate, tissue velocity imaging, and endothelial function for early detection of cardiovascular involvement in patients with beta-thalassemia. Annals of pediatric cardiology 2017;10(2): 158-66.

[13] Galderisi M, Cosyns B, Edvardsen T, et al. Standardization of adult transthoracic echocardiography reporting in agreement with recent chamber quantification, diastolic function, and heart valve disease recommendations: an expert consensus document of the European Association of Cardiovascular Imaging. European heart journal cardiovascular Imaging 2017;18(12): 1301-10.

[14] Kremastinos D, Toutouzas P, Vyssoulis G, Venetis C, Avgoustakis D. Iron overload and left ventricular performance in beta thalassemia. Acta cardiologica 1984;39(1): 29-40.

[15] Garceau P, Nguyen ET, Carasso S, et al. Quantification of myocardial iron deposition by two-dimensional speckle tracking in patients with beta-thalassaemia major and Blackfan-Diamond anaemia. Heart 2011; 97(5): 388-93.

[16] Karamanou AG, Hamodraka ES, Vrakas SC, et al. Assessment of left ventricular and atrial diastolic function using twodimensional (2D) strain imaging in patients with betathalassemia major. Eur J Haematol 2014; 92(1): 59-65.

[17] Parsaee M, Akiash N, Azarkeivan A, et al. The correlation between cardiac magnetic resonance $T 2^{*}$ and left ventricular global longitudinal strain in people with betathalassemia. Echocardiography 2018; 35(4): 438-44.

[18] Pizzino F, Meloni A, Terrizzi A, et al. Detection of myocardial iron overload by two-dimensional speckle tracking in patients with beta-thalassaemia major: a combined echocardiographic and T2* segmental CMR study. The International Journal of Cardiovascular Imaging 2018; 34(2): 263-71. 